\title{
Quais os efeitos da natação para crianças e adolescentes? Revisão sistemática de literatura
}

What are the effects of swimming for children and adolescents? Systematic review of literature

\author{
Clara Knierim Correia ${ }^{1, *}$ \\ Eduardo Henrique Zanella de Arruda ${ }^{2}$ \\ Arthur Dutra dos Santos ${ }^{1}$ \\ Renata Maba Gonçalves Wamosy \\ Hélio Roesler ${ }^{1}$ \\ Suzana Matheus Pereira ${ }^{1}$
}

\begin{abstract}
Resumo
Objetivo: Verificar os efeitos fisiológicos, psicológicos e biomecânicos da prática de natação em crianças e adolescentes, utilizando uma revisão sistemática de literatura. Método: De acordo com a declaração PRISMA os estudos foram identificados por meio de quatro bases de dados (Web of Science, Sport Discus, Science Direct e Pubmed). Os termos utilizados para buscas foram relacionadas as crianças/adolescentes (child, preschool, pediatric, adolescent), natação (swim, aquatic exercise) e aos desfechos (physiologic, psychologic, biomechanics). $O$ processo de busca seguiu as recomendações da estratégia PICO. Resultados: A busca retornou 727 estudos, destes, apenas 11 foram incluídos na revisão. Os estudos comparam a natação com outros esportes, jovens saudáveis e sedentários. Em relação a amostra, há predomínio de meninos $(n=187)$ e de faixa etária entre seis e 20 anos. A maior parte dos estudos investigou aspectos fisiológicos, predominando a capacidade pulmonar e cardiorrespiratória. Conclusão: A prática de natação parece promover aumento no $\mathrm{VO}_{2}$ máximo e função pulmonar, além de maior autoeficácia, qualidade do sono e sociabilização em crianças e adolescentes.
\end{abstract}

Palavras-chave: natação, crianças, adolescentes, revisão sistemática.

\begin{abstract}
Objective: To verify the physiological, psychological and biomechanical effects of swimming practice in children and adolescents, using as systematic review of the literature. Methods: According to the PRISMA statement, the studies were identified through four databases (Web of Science, SportDiscus, Science Direct and Pubmed). The terms used for searches were child/adolescent (child, preschool, pediatric, adolescent), swim (aquatic exercise) and outcomes (physiologic, psychologic, biomechanics). The search process followed the recommendations of the PICO strategy. Results: The search returned 727 studies, of which only 11 were included in the review. The studies compare swimming with other sports, healthy and sedentary youth. In relation to the sample, there is a predominance of boys $(n=187)$ and age group between six and 20 years. Most of the studies investigated physiological aspects, predominating pulmonary and cardiorespiratory capacity. Conclusion: Swimming practice seems to promote an increase in maximal $\mathrm{VO}_{2}$ and pulmonary function, in addition to greater self-efficacy, sleep quality and socialization in children and adolescents.
\end{abstract}

Keywords: swimming, children, adolescents, systematic review.
Afiliação dos autores

${ }^{1}$ Universidade do Estado de Santa Catarina, Florianópolis, Santa Catarina, Brasil.

${ }^{2}$ Universidade Federal de Santa Catarina, Florianópolis, Santa

Catarina, Brasil.

${ }^{\star}$ Autor correspondente

Universidade do Estado de Santa Catarina, Departamento de Educação Física. Laboratório de Pesquisas em Biomecânica Aquática. Rua Pascoal Simone, 358, Coqueiros, CEP: 88080-350. Florianópolis, Santa Catarina, Brasil. e-mail: clara.kc@hotmail.com

Conflito de interesses

Os autores declararam não haver conflito de interesses.

Processo de arbitragem

Recebido: 31/12/2018 Aprovado: 06/03/2019 


\section{Introdução}

As elevadas taxas de mortes por afogamentos em lagos, rios, mares e piscinas são consideradas casos de Saúde Pública em diferentes locais no mundo ${ }^{1,2}$. Partindo dessa premissa, a natação se torna fundamental, atuando na prevenção contra afogamentos ${ }^{3}$. Além de preconizar a segurança aquática, a natação é considerada um esporte popular e com inúmeros benefícios. Por ser ensinada desde a primeira infância e por não haver uma faixa etária máxima para praticar, os benefícios da natação podem perdurar ao longo da vida. Em populações especiais, a natação também contribui positivamente ${ }^{4-6}$. Neste sentido, em pacientes com comprometimento motor, a natação promove independência, resultando em uma melhor qualidade de vida ${ }^{4,6}$. Em crianças asmáticas, a natação é considerada a prática de atividade física mais recomendada, visto seus benefícios físicos, fisiológicos e psicológicos ${ }^{5}$.

Recentes revisões de literatura têm dado destaque apenas para a natação competitiva ${ }^{7-9}$. Tais achados investigam os atletas em aspectos biomecânicos ${ }^{8}$ ou fisiológicos ${ }^{7,9}$ e que estejam estritamente vinculadas a uma performance de excelência. Neste contexto, diferentes tipos de treinamento ${ }^{7,9}$ e protocolos de análises para atletas ${ }^{7,8}$ têm sido evidenciadas, buscando aperfeiçoar o nível técnico para obter o melhor eficiência durante as competições. Em atletas, há uma predominância de se estudar variáveis que influenciam o rendimento durante o nado craw $P^{\beta}$, tanto no período de treinamento, quanto no período competitivo.

Visto a relevância e importância das pesquisas da natação em atletas, até o momento foi identificada apenas uma revisão sistemática de literatura com meta-análise que analisou os efeitos da natação em indivíduos saudáveis ${ }^{3}$. Os resultados indicam que a natação pode promover melhoras fisiológicas, principalmente na aptidão cardiorrespiratória e composição corporal. Entretanto, não foram encontradas revisões sistemáticas que sumarizem os efeitos fisiológicos, psicológicos e biomecânicos da natação em crianças e adolescentes. Resultados desta natureza podem direcionar diferentes áreas das Ciências do exercício e esporte, como prescrição, fisiologia e psicologia do esporte e do exercício, por exemplo.

Além disso, por ser uma prática considerada sazonal, em que o número maior de praticantes ocorre nas estações mais quentes ${ }^{10}$, enaltecer os benefícios da prática regular da natação pode aumentar o número de adeptos, além de contribuir para maior assiduidade daqueles que já praticam. Diante do exposto, o objetivo deste estudo foi verificar os efeitos fisiológicos, psicológicos e biomecânicos da prática de natação em crianças e adolescentes, por meio de uma revisão sistemática de literatura.

\section{Métodos}

Esta revisão foi elaborada de acordo com a Declaração PRISMA - Preferred Reporting Items for Systematic reviews and Meta-Analyses ${ }^{11}$.

\section{Estratégias de Busca}

Foi realizada a busca nas bases eletrônicas de dados: Web of Science, Science Direct, SPORT Discuse Pubmed. Os termos utilizados seguiram as recomendações do Medical Subject Headings (MeSH) e compreendem termos que representam crianças e adolescentes; natação e efeitos fisiológicos, psicológicos e biomecânicos, conforme o Quadro 1.

Quadro 1

Descritores utilizados nas buscas.

\begin{tabular}{|c|c|}
\hline Blocos & Descritores \\
\hline $\begin{array}{c}\text { \#1 Crianças e } \\
\text { adolescentes }\end{array}$ & child OR preschool OR pediatric OR adolescent \\
\hline \#2 Natação & swim OR "aquatic exercise" \\
\hline \#3 Efeitos & $\begin{array}{c}\text { physiological OR psychological OR } \\
\text { biomechanics }\end{array}$ \\
\hline Combinação & \#1 AND \#2 AND \#3 \\
\hline
\end{tabular}

A busca nas bases ocorreu em novembro de 2018 e não foi realizado nenhum recorte temporal.

\section{Critérios de Elegibilidade}

Os critérios de elegibilidade foram estabelecidos de acordo com a estratégia PICO ${ }^{11,12}$. Sendo assim:

População: Crianças e adolescentes, com idade compreendida de 0 meses a 19 anosou com a média de idade dos participantes compreendida entre 0 meses a 19 anos;

Intervenção: natação ou exercícios aquáticos;

Comparação: Pré e pós intervenção, população de não nadadores ou após um longo período de prática de natação;

Desfechos: Qualquer variável de caráter psicológico, biomecânico ou fisiológico.

Design do estudo: Estudo de caso, Experimental, quaseexperimental, longitudinal ou transversal. Optou-se por abranger estudos de corte transversal visto que algumas pesquisas incluem participantes que praticam natação por mais de um ano, além de comparações com outras modalidades esportivas.

Foram incluídos estudos disponíveis apenas no idioma inglês. Estudos não disponíveis na integra, teses, dissertações e anais de congresso foram considerados critérios de exclusão.

\section{Seleção dos Estudos}

O processo de busca foi realizado independentemente por dois pesquisadores (CKC; RMGW), com base nos critérios de elegibilidade estabelecidos. A inclusão dos estudos foi determinada pelas etapas de seleção dos títulos, excluindo-se os duplicados, que foram acurados para a leitura dos resumos. Após a seleção dos resumos, os artigos foram analisados na integra, e incluídos aqueles que atendessem aos critérios determinados. Em caso de divergência de estudos, um terceiro autor (EHZA) foi solicitado para opinião final.

\section{Extração e síntese dos dados}

A extração e síntese dos dados foram realizadas a partir de três categorias: 1) Característica dos estudos: Ano, design do estudo, comparação e protocolo de intervenção; 2) Característica da amostra: Número de participantes, sexo, idade e experiência prévia com natação; 3) Principais resultados: Desfecho (fisiológico, psicológico de biomecânico), variáveis analisadas e principais resultados identificados, sendo definidos como aquelas que apresentaram diferença significante.

\section{Risco de viés}

O risco de viés foi avaliado por meio do instrumento proposto por Higgins et al. ${ }^{13}$ e traduzido por Carvalho e Grande $^{14}$. O instrumento propõe avaliar a qualidade dos estudos incluídos na revisão de acordo com seis aspectos: geração da sequência aleatória; ocultação de alocação; cegamento dos participantes e profissionais; cegamento de avaliadores de desfecho; desfechos incompletos; relato de desfecho seletivo; outras fontes de viés.

Os resultados dos instrumentos indicam se o estudo apresenta baixo risco de viés, risco de viés incerto ou alto risco de viés. As análises foram realizadas separadamente por dois pesquisadores (CKC; RMGW) e, em caso de divergência de análise, um terceiro pesquisador (EHZA) foi consultado para avaliação final.

\section{Resultados}

Após o processo de busca, realizado no dia 10 de dezembro de 2018, foram identificados 727 artigos, dos quais sete eram duplicados, resultando em 720 estudos para leitura do título. Destes, 82 artigos foram selecionados por meio do título e, após a leitura do resumo, 36 artigos foram escolhidos para a leitura na íntegra. Após o processo de elegibilidade, 11 artigos foram incluídos para a análise final, conforme representado na Figura 1.

A síntese dos dados dentre as três categorias de análises estão apresentas no Quadro 2 e serão descritas a seguir.

\section{Característica dos estudos}

O primeiro estudo publicado buscando verificar os efeitos da natação em crianças e/ou adolescentes foi no ano de $1980^{15}$. Os ápices das publicações foram nos anos de $2007^{16,17}$ e $2018^{18,19}$, 
Quadro 2. Síntese dos resultados dos 11 estudos incluídos na revisão, de acordo com as características dos estudos, amostra e principais resultados.

\begin{tabular}{|c|c|c|c|c|c|c|c|c|c|c|}
\hline \multirow[b]{2}{*}{ Referência } & \multirow[b]{2}{*}{ Ano } & \multicolumn{3}{|c|}{ Característica da amostra } & \multicolumn{3}{|c|}{ Característica dos estudos } & \multirow[b]{2}{*}{ Desfecho } & \multirow[b]{2}{*}{$\begin{array}{l}\text { Variáveis } \\
\text { (principais) }\end{array}$} & \multirow[b]{2}{*}{$\begin{array}{l}\text { Principais } \\
\text { resultados }\end{array}$} \\
\hline & & $n(M / F)$ & Idade & $\begin{array}{c}\text { Experiência } \\
\text { prévia com } \\
\text { natação }\end{array}$ & $\begin{array}{l}\text { Design do } \\
\text { estudo }\end{array}$ & Comparação & $\begin{array}{c}\text { Protocolo de } \\
\text { intervenção ou } \\
\text { coleta de dados }\end{array}$ & & & \\
\hline $\begin{array}{l}\text { Atamturk e } \\
\text { Atamturk }^{18}\end{array}$ & 2018 & Um menino & 6 anos & Não & $\begin{array}{l}\text { Relato de } \\
\text { caso }\end{array}$ & $\begin{array}{l}\text { Antes e após } \\
\text { o protocolo }\end{array}$ & $\begin{array}{l}8 \text { semanas, } 1 \text { vez } \\
\text { por semana por } \\
45 \text { minutos }\end{array}$ & Psicológico & $\begin{array}{l}\text { Psicológico e } \\
\text { emocional }\end{array}$ & $\begin{array}{c}\text { Melhora da } \\
\text { qualidade do } \\
\text { sono, } \\
\text { socialização e } \\
\text { relaxamento }\end{array}$ \\
\hline Couto et al. ${ }^{23}$ & 2015 & $\begin{array}{l}\text { Asmáticos: } \\
10(7 / 3) \\
\text { Não } \\
\text { asmáticos: } \\
(3 / 9)\end{array}$ & $\begin{array}{l}17 \pm 2,9 \\
\text { anos }\end{array}$ & $\begin{array}{l}\text { Nadadores } \\
\text { de elite } \\
\text { (período } \\
\text { médio de } 9 \\
\text { anos de } \\
\text { competição) }\end{array}$ & Experimental & $\begin{array}{c}\text { Nadadores } \\
\text { asmáticos e } \\
\text { não } \\
\text { asmáticos }\end{array}$ & $\begin{array}{c}\text { Uma visita } \\
\text { composta por três } \\
\text { etapas } \\
\text { (treinamento } \\
\text { aeróbio, } \\
\text { anaeróbio e } \\
\text { moderado/intenso) }\end{array}$ & Fisiológico & $\begin{array}{l}\text { Função pulmonar, } \\
\text { teste de bronco- } \\
\text { provacação e } \\
\text { temperatura do ar } \\
\text { exalado }\end{array}$ & $\begin{array}{c}\text { A temperatura } \\
\text { do ar exalado } \\
\text { aumentou em } \\
\text { ambos os } \\
\text { grupos }\end{array}$ \\
\hline $\begin{array}{l}\text { D'Ascenzi et } \\
\text { al. }^{22}\end{array}$ & 2017 & $\begin{array}{l}\text { Controle: } \\
37 \text { meninos } \\
\text { Atletas: } 57 \\
\text { meninos }\end{array}$ & $\begin{array}{l}10,8 \pm 0,2 \\
\text { anos }\end{array}$ & $\begin{array}{l}\text { Ativos, em } \\
\text { começo de } \\
\text { temporada }\end{array}$ & Prospectivo & $\begin{array}{l}\text { Nadadores e } \\
\text { controle }\end{array}$ & $\begin{array}{l}\text { Cinco meses: uma } \\
\text { vez por dia, por } 5 \\
\text { a } 6 \text { vezes por } \\
\text { semana }\end{array}$ & Fisiológico & $\begin{array}{l}\text { Morfologia } \\
\text { cardíaca }\end{array}$ & $\begin{array}{l}\text { A natação } \\
\text { promove } \\
\text { remodelamento } \\
\text { do VD, com } \\
\text { função } \\
\text { cardíaca } \\
\text { preservada } \\
\end{array}$ \\
\hline $\begin{array}{l}\text { Jürimäe et } \\
\text { al. }^{17}\end{array}$ & 2007 & 29 meninos & $\begin{array}{c}13 \pm 1,8 \\
\text { anos }\end{array}$ & $\begin{array}{c}\text { Formação de } \\
3 \text { anos } \\
\text { (média de } \\
\text { treinamento } \\
\text { de } 8 \text { horas } \\
\text { por semana) }\end{array}$ & Transversal & $\begin{array}{l}\text { Nadadores } \\
\text { pré-púberes } \\
\text { e púberes }\end{array}$ & $\begin{array}{c}\text { Durante o período } \\
\text { de teste, o volume } \\
\text { médio semanal de } \\
\text { treinamento foi de } \\
19,4 \pm 5,5 \mathrm{~km}\end{array}$ & Fisiológico & $\begin{array}{c}\text { Capacidade } \\
\text { cardiorrespiratória }\end{array}$ & $\begin{array}{l}\mathrm{VO}_{2} \text { pico foi } \\
\text { maior em } \\
\text { ambos os } \\
\text { grupos de } \\
\text { nadadores }\end{array}$ \\
\hline Latt et al. ${ }^{21}$ & 2009 & 26 meninas & $12-14$ anos & $\begin{array}{l}\text { Formação de } \\
3 \text { anos } \\
\text { (média de } \\
\text { treinamento } \\
\text { de } 6 \text { horas } \\
\text { por semana) }\end{array}$ & Prospectivo & $\begin{array}{c}\text { Antes e } \\
\text { depois do } \\
\text { treinamento } \\
\text { de natação }\end{array}$ & $\begin{array}{l}\text { Dois anos de } \\
\text { acompanhamento }\end{array}$ & $\begin{array}{l}\text { Biomecânico } \\
\text { e fisiológico }\end{array}$ & $\begin{array}{c}\text { Parâmetros } \\
\text { biomecânicos, } \\
\text { capacidade } \\
\text { cardiorrespiratória }\end{array}$ & $\begin{array}{l}\text { A velocidade, } \\
\text { braçadas e } \\
\text { valor } \\
\text { energético } \\
\text { aumentaram } \\
\text { na terceira } \\
\text { avaliação, } \mathrm{VO}_{2} \\
\text { aumentou após } \\
\text { a segunda } \\
\text { avaliação }\end{array}$ \\
\hline $\begin{array}{l}\text { Mickleborough } \\
\text { et al. }^{25}\end{array}$ & 2008 & $30(15 / 15)$ & $\begin{array}{l}18 \pm 1,6 \\
\text { anos }\end{array}$ & $\begin{array}{l}\text { Atletas de } \\
50-100 \\
\text { metros, } 200- \\
400 \text { metros e } \\
800-1500 \\
\text { metros }\end{array}$ & Experimental & $\begin{array}{l}\text { Antes e após } \\
\text { o protocolo }\end{array}$ & $\begin{array}{l}12 \text { semanas de } \\
\text { treinamento - } 3 \\
\text { grupos: } \\
\text { 1) natação } \\
\text { 2) natação + TMI } \\
\text { 3) natação + } \\
\text { placebo de TMI }\end{array}$ & Fisiológico & $\begin{array}{l}\text { Função pulmonar, } \\
\text { força e } \\
\text { capacidade da } \\
\text { musculatura } \\
\text { respiratória }\end{array}$ & $\begin{array}{c}\text { Todos os } \\
\text { grupos tiveram } \\
\text { aumento dos } \\
\text { parâmetros de } \\
\text { função } \\
\text { pulmonar e } \\
\text { FMR após o } \\
\text { protocolo, e ao } \\
\text { comprar os } \\
\text { grupos o grupo } \\
\text { (2) teve } \\
\text { melhores } \\
\text { resultados } \\
\end{array}$ \\
\hline $\begin{array}{l}\text { Navarro e } \\
\text { Granell }^{19}\end{array}$ & 2018 & $123(68 / 55)$ & $12-20$ anos & $\begin{array}{l}\text { Atletas de } \\
\text { alto nível }\end{array}$ & Transversal & $\begin{array}{l}\text { Modalidades: } \\
\text { atletismo, } \\
\text { natação e } \\
\text { triathlon, } \\
\text { além de faixa } \\
\text { etária e sexo }\end{array}$ & $\begin{array}{c}\text { Uma visita durante } \\
\text { o período } \\
\text { competitivo, } 3 \text { dias } \\
\text { antes das } \\
\text { avaliações } \\
\text { ocorreram } \\
\text { diminuição de } \\
\text { intensidade }\end{array}$ & Fisiológico & $\begin{array}{c}\text { Capacidade } \\
\text { cardiorrespiratória }\end{array}$ & $\begin{array}{c}\mathrm{VO}_{2} \text { máximo foi } \\
\text { maior na } \\
\text { natação, do } \\
\text { que nos outros } \\
\text { grupos } \\
\text { (triathlon e } \\
\text { atletismo) }\end{array}$ \\
\hline $\begin{array}{l}\text { Silvestri et } \\
\text { al. }^{20}\end{array}$ & 2013 & $34(28 / 6)$ & $7-20$ anos & $\begin{array}{l}\text { Acúmulo de } \\
\text { no mínimo } \\
400 \text { horas de } \\
\text { treinamento }\end{array}$ & Transversal & $\begin{array}{c}\text { Grupo } \\
\text { controle (não } \\
\text { nadadores) }\end{array}$ & $\begin{array}{l}\text { Uma única visita, } \\
\text { não informando } \\
\text { em que período } \\
\text { de treinamento os } \\
\text { participantes } \\
\text { estavam. }\end{array}$ & Fisiológico & $\begin{array}{c}\text { Sintomas } \\
\text { respiratórios, } \\
\text { função pulmonar } \\
\text { e teste de bronco- } \\
\text { provocação }\end{array}$ & $\begin{array}{l}\text { Capacidade } \\
\text { vital forçada foi } \\
\text { positivamente } \\
\text { associado com } \\
\text { a idade }\end{array}$ \\
\hline $\begin{array}{l}\text { Triposkiadis et } \\
\text { al. }^{16}\end{array}$ & 2007 & $\begin{array}{l}\text { Controle: } \\
7(1 / 6) \\
\text { Nadadores: } \\
25(15 / 10)\end{array}$ & $\begin{array}{c}\text { nadadores: } \\
11,9 \pm 1,6 \\
\text { anos } \\
\text { controle: } \\
11,3 \pm 0,6 \\
\text { anos }\end{array}$ & $\begin{array}{c}\text { Grupo } \\
\text { nadadores: } \\
12 \text { a } 14 \\
\text { horas } \\
\text { semanais } \\
\text { nos últimos } 4 \\
\text { anos } \\
\text { controle: } \\
\text { ativos } \\
\text { fisicamente } \\
\end{array}$ & Transversal & $\begin{array}{c}\text { Nadadores e } \\
\text { não } \\
\text { nadadores }\end{array}$ & $\begin{array}{l}\text { Uma única visita, } \\
\text { não informando } \\
\text { em que período } \\
\text { de treinamento os } \\
\text { participantes } \\
\text { estavam }\end{array}$ & Fisiológico & $\begin{array}{l}\text { Modelagem } \\
\text { cardíaca }\end{array}$ & $\begin{array}{c}\text { A natação } \\
\text { promoveu } \\
\text { remodelamento } \\
\text { cardíaco }\end{array}$ \\
\hline $\begin{array}{l}\text { Vaccaro et } \\
\text { al. }^{15}\end{array}$ & 1980 & 12 meninos & $\begin{array}{c}15,1 \pm 1,7 \\
\text { anos }\end{array}$ & $\begin{array}{c}\text { Atletas por } \\
\text { pelo menos } 6 \\
\text { anos } \\
\text { consecutivos, } \\
\text { treinamento } \\
\text { de } 4 \text { dias por } \\
\text { semana } \\
\end{array}$ & Transversal & $\mathrm{N} / \mathrm{A}$ & $\begin{array}{l}\text { Duas visitas } \\
\text { durante o ápice do } \\
\text { período } \\
\text { competitivo }\end{array}$ & Fisiológico & $\begin{array}{l}\text { Composição } \\
\text { corporal, força } \\
\text { muscular, função } \\
\text { pulmonare } \\
\text { capacidade } \\
\text { aeróbica }\end{array}$ & $\begin{array}{l}\text { Capacidade } \\
\text { vital forçada } \\
\text { (espirometria) } \\
\text { foi maior do } \\
\text { que os valores } \\
\text { normativos. }\end{array}$ \\
\hline Weiss et al. ${ }^{24}$ & 1998 & $24(18 / 6)$ & 6,2 anos & $\begin{array}{l}\text { Média de } 9,5 \\
\text { semanas de } \\
\text { aulas de } \\
\text { natação }\end{array}$ & Experimental & $\begin{array}{c}\text { Grupos: } \\
\text { 01: Controle, } \\
02: \\
\text { peermastery } \\
\text { 03: } \\
\text { peercoping } \\
\end{array}$ & $\begin{array}{c}\text { Nove dias de } \\
\text { aulas }\end{array}$ & Psicológico & $\begin{array}{l}\text { Medo de água e } \\
\text { autoeficácia }\end{array}$ & $\begin{array}{c}\text { Medo de água } \\
\text { reduziu nos } \\
\text { grupos } 02 \text { e } 03 \\
\text { e autoeficiácia } \\
\text { aumentou nos } \\
\text { grupos } 02 \text { e } 03 \\
\end{array}$ \\
\hline
\end{tabular}


com dois estudos cada. Em relação ao design dos estudos incluídos nesta revisão, cinco são de caráter transversal ${ }^{15-17,19,20}$, três são do tipo prospectivo ${ }^{21-23}$, dois de cunho experimental ${ }^{24,25}$ e um relato de caso ${ }^{18}$

Em se tratando dos tipos de comparações, dois estudos comparam a natação com outras modalidades esportivas ${ }^{17,19}$, três comparam com grupo controle (sedentários ou ativos fisicamente $)^{16,20,22}$, três comparam antes e depois do protocolo experimental $^{18,24,25}$ e apenas um estudo comparou o efeito da natação em crianças com diagnóstico de asma ${ }^{23}$. Dos 11 estudos, apenas dois verificam desfechos após longos períodos de prática de natação ${ }^{15,21}$.

Os protocolos de intervenção ou de coleta de dados se mostraram distintos entre os estudos. Dentre os protocolos experimentais, os estudos apresentaram intervenção de uma única visita $^{16,17,19,20,23}$ a 12 semanas $^{25}$. A duração dos estudos prospectivos variaram de cinco meses ${ }^{22}$ a dois anos ${ }^{21}$. Dentre os estudos de corte transversal, dois estudos ${ }^{16,20}$ não informaram em que fase do treinamento os atletas estavam.

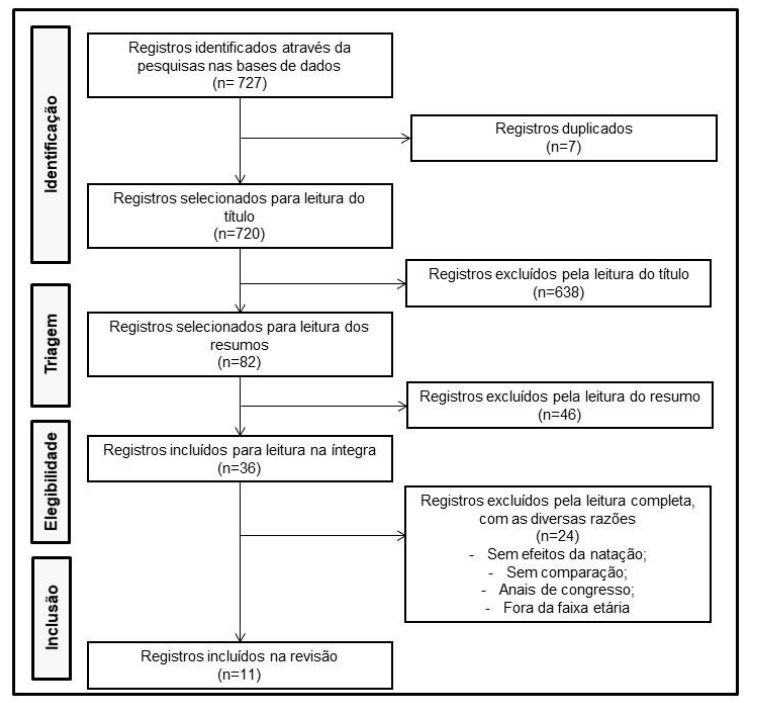

Figura 1. Fluxograma do processo de seleção dos estudos.

\section{Características dos participantes}

No total, 427 crianças e adolescentes, sendo 187 meninos e 124 meninas foram investigadas. Dois estudos não informaram o gênero dos sujeitos ${ }^{22,23}$. O número de participantes variou de $u^{18}$ a $123^{19}$. A faixa etária dos participantes foi entre seis ${ }^{18}$ e 20 anos $^{19}$. Dos 11 estudos incluídos, apenas dois não incluíram em suas amostras nadadores competitivos ${ }^{18,24}$. Os critérios de inclusão dos participantes dos estudos se diferenciaram por recrutarem através de horas semanais ${ }^{16,20,24}$ ou anos $^{15,17,21,23}$ de treinamento ou prática de natação. Apenas um estudo incluiu participante sem experiência prévia com natação ${ }^{18}$.

\section{Principais resultados identificados}

Dos 11 estudos incluídos, nove abordaram desfechos fisiológicos ${ }^{15-17,19-23,25}$, dois psicológicos ${ }^{18,24}$ e um biomecânico ${ }^{21}$. Variáveis relacionadas à função pulmonar foram as mais estudadas $^{20,23,25}$, seguido de variáveis cardíacas ${ }^{16,22}$ e capacidade cardiorrespiratória ${ }^{15,17,21}$. As demais variáveis estão apresentadas na figura 1 , e apenas foram investigadas uma única vez.

De acordo com o risco de viés, todos os estudos apresentaram baixo risco de viés (Quadro 3). Aspectos relacionados ao cegamento dos participantes foram indicados pelos pesquisadores como baixo risco de viés, visto que este componente não afetaria os desfechos dos estudos ${ }^{26}$.

\section{Discussão}

A corrente revisão teve como objetivo verificar os efeitos fisiológicos, psicológicos e biomecânicos da prática de natação em crianças e adolescentes, por meio de uma revisão sistemática de literatura. Dos resultados identificados, observouse que a prática de natação pode promover melhoras na capacidade cardiorrespiratória (aumento do volume máximo de oxigênio - $\left.\mathrm{VO}_{2}\right)^{17,19,21}$, remodelamento cardíaco ${ }^{16,22}$ e função pulmonar (capacidade vital forçada) ${ }^{15,20,25}$. Em se tratando de aspectos psicológicos, a natação parece aumentar a autoeficácia $^{24}$, reduzir o medo de água ${ }^{24}$, melhora a qualidade do sono e sociabilização ${ }^{18}$.

Quadro 3. Análise dos critérios para avaliação dos riscos de viés de cada estudo selecionado.

\begin{tabular}{|c|c|c|c|c|c|c|c|}
\hline Referência & $\begin{array}{l}\text { Geração de } \\
\text { sequência } \\
\text { aleatória }\end{array}$ & $\begin{array}{l}\text { Ocultação de } \\
\text { alocação }\end{array}$ & $\begin{array}{l}\text { Cegamento de } \\
\text { participantes e } \\
\text { profissionais }\end{array}$ & $\begin{array}{l}\text { Cegamento de } \\
\text { avaliadores de } \\
\text { desfecho }\end{array}$ & $\begin{array}{l}\text { Desfechos } \\
\text { incompletos }\end{array}$ & $\begin{array}{l}\text { Relato de } \\
\text { desfecho } \\
\text { seletivo }\end{array}$ & $\begin{array}{c}\text { Outras } \\
\text { fontes de } \\
\text { viés }\end{array}$ \\
\hline $\begin{array}{l}\text { Atamturk e } \\
\text { Atamturk }^{18}\end{array}$ & - & - & + & + & + & $?$ & + \\
\hline Couto et al. ${ }^{23}$ & - & - & + & + & + & + & + \\
\hline D'Ascenzi et al. $^{22}$ & - & - & + & + & + & $?$ & + \\
\hline Jürimäe et al. ${ }^{17}$ & - & - & + & + & + & + & + \\
\hline Latt et al. ${ }^{21}$ & - & - & + & + & $?$ & $?$ & + \\
\hline Mickleborough et al. ${ }^{25}$ & - & - & + & + & $?$ & $?$ & + \\
\hline Navarro e Granell ${ }^{19}$ & - & - & + & + & + & $?$ & + \\
\hline Silvestri et al. ${ }^{20}$ & - & - & + & + & + & $?$ & + \\
\hline Triposkiadis et al. $^{16}$ & - & - & + & + & $?$ & $?$ & + \\
\hline Vaccaro et al. $^{15}$ & - & - & + & + & $?$ & $?$ & - \\
\hline Weiss et al. ${ }^{24}$ & - & - & + & + & + & ? & + \\
\hline
\end{tabular}

Legenda: +: baixo risco de viés; ?: risco de viés incerto; -: alto risco de viés.

Principalmente no que tange aos desfechos fisiológicos, os nossos resultados foram semelhantes aos encontrados na revisão de Lahart e Metsois ${ }^{3}$. Ambos os estudos identificaram que a prática da natação aumenta $\circ \mathrm{VO}_{2}$ e função pulmonar (capacidade vital forçada). Além dos parâmetros cardiorrespiratórios e função pulmonar, a força muscular respiratória pode melhorar após protocolos de natação em crianças. Esses resultados podem estar associados ao fato da natação possuir característica predominantemente aeróbia, contribuindo para maiores demandas pulmonares. Tais demandas estão estritamente ligadas aos fatores fisiológicos da respiração, como as trocas gasosas e diminuição da resistência das vias aéreas, por exemplo ${ }^{5}$.

No que concerne os efeitos psicológicos, foram poucos os estudos que abordaram essa temática. Variáveis psicológicas são fundamentais, pois podem direcionar a estratégia de aderência para um maior número de adeptos à prática da natação. Neste contexto, crianças praticantes de natação apresentam melhores capacidades físicas ${ }^{27}$ e que estão associadas a uma maior autoeficácia ${ }^{24}$. Visto que a natação aumenta a autoeficácia ${ }^{24}$, é possível especular que o praticante consiga se manter no esporte por longo período de tempo. Além disso, Matias et al. ${ }^{28}$ contribuem indicando que adolescentes que apresentam maiores níveis de autoeficácia estão associados a um estilo de vida mais saudável.

Dentro os aspectos biomecânicos identificados, percebe-se que os mesmos são estudados em população de atletas. Este fato pode ser explicado no sentido de que a natação competitiva busca a alta performance técnica. Esses parâmetros podem ser avaliados principalmente por meio de análises cinemáticas ${ }^{21,29}$, verificando aspectos espaço-temporais, como a frequência de braçada. Levando em consideração crianças e adolescentes praticantes de natação, a biomecânica deve ser estudada como forma ensino da técnica, buscando reduzir as lesões atraumáticas causadas por esportes com gestos repetitivos ${ }^{30}$. 
A variabilidade de desfechos e populações dos estudos encontrados pode ser considerada uma limitação na análise comparativa dos resultados. A maioria dos estudos revisados incluem crianças e adolescentes considerados atletas, em treinamento, em início ou meio de temporada, o que dificulta a extrapolação desses dados para a população pediátrica geral. Ainda assim, as adaptações e resultados encontrados nas crianças atletas podem fornecer informações importantes sobre os benefícios da natação.

Sugerem-se novos estudos com o propósito de comparar a natação com outras modalidades esportivas, incluir crianças e adolescentes sem experiência prévia com natação ou esportes aquáticos. Além disso, também torna-se necessário investigar outras variáveis psicológicas como estados de humor e motivação, além de diferentes parâmetros biomecânicos, como cinemática dos diferentes nados.

\section{Conclusão}

A prática de natação proporciona efeitos benéficos fisiológicos, biomecânicos e psicológicos como aumento no $\mathrm{VO}_{2}$ máximo e parâmetros de função pulmonar, além de maior autoeficácia, qualidade do sono e sociabilização em crianças e adolescentes

\section{Referências}

1. Lin C-Y, Wang Y-F, Lu T-H, Kawach I. Unintentional drowning mortality, by age and body of water: an analysis of 60 countries. Inj Prev. 2015;21(e1):e43-50.

2. Leavy JE, Crawford G, Portsmouth L, Jancey J, Leaversuch F, Nimmo L, et al. Recreational Drowning Prevention Interventions for Adults, 1990-2012: A Review. J Community Health. 2015;40(4):725-35.

3. Lahart IM, Metsios GS. Chronic Physiological Effects of Swim Training Interventions in Non-Elite Swimmers: A Systematic Review and MetaAnalysis. Sport Med. 2018;48(2):337-59.

4. Corte M, Da Silva R, Jacó De Oliveira R. Efeitos da natação sobre a independência funcional de pacientes com lesão medular. Rev Bras Med Esporte. 2005;11(4):251-256.

5. Corazza ST, Silva MCR da, Paulus LD, Trindade CPP, Vidor DM. Asma infantil - Esclarecimentos e uma proposta de intervenção motora física e funcional. Pensar a Prática. 2016:19(1):232-244.

6. Getz M, Hutzler Y, Vermeer $A$. The relationship between aquatic independence and gross motor function in children with neuro-motor impairments. Adapt Phys Act Q. 2006;23(4):339-55.

7. Costa MJ, Bragada JA, Marinho, ANTJ, Barbosa TM. Longitudinal interventions in elite swimming: asystematic review based on energetics, biomechanics, and performance. J Strength Cond Res. 2012;26(6):2006biomec.

8. Martens J, Figueiredo P, Daly D. Electromyography in the four competitive swimming strokes: A systematic review. J Electromyogr Kinesiol. 2015;25(2):273-91

9. Crowley E, Harrison AJ, Lyons M. The Impact of Resistance Training on Swimming Performance: A Systematic Review. Sport Med. 2017:47(11):2285-307.

10. Tucker $P$, Gilliland J. The effect of season and weather on physical activity: A systematic review. Public Health. 2007;121(12):909-22.

11. Moher D, Shamseer L, Clarke M, Ghersi D, Liberati A, Petticrew M, et al.
Preferred reporting items for systematic review and meta-analysis protocols (PRISMA-P) 2015 statement. Syst Rev. 2015;1(4):1-9.

12. Santos CMC, Pimenta CAM, Nobre MRC. A estratégia PICO para a construção da pergunta de pesquisa e busca de evidências. Rev LatinoAmericana Enfer. 2007;15(3):508-511.

13. Higgins JPT, Altman DG, Gøtzsche PC, Jüni $P$, Moher D, Senior ADO, et al. The Cochrane Collaboration's tool for assessing risk of bias in randomised trials. BMJ Open. 2011;343:1-9.

14. Carvalho APV de, Silva V, Grande AJ. Avaliação do risco de viés de ensaios clínicos randomizados pela ferramenta da colaboração Cochrane. Diagnóstico Trat . 2013:1(1):38-44.

15. Vaccaro P, Clarke DH, Morris AF. Physiological characteristics of young well-trained swimmers. Eur J Appl Physiol Occup Physiol. 1980;44(1):616.

16. Triposkiadis F, Ghiokas S, Skoularigis I, Kotsakis A, Giannakoulis I, Thanopoulos V. Cardiac adaptation to intensive training in prepubertal swimmers. Eur J Clin Invest. 2002;32(1):16-23.

17. Jürimäe $J$, Haljaste $K$, Cicchella $A$, Lätt $E$, Purge $P$, Leppik $A$, et al. Analysis of Swimming Performance from Physical, Physiological, and Biomechanical Parameters in Young Swimmers. Pediatr Exerc Sci. 2007;19(1):70-81.

18. Atamturk $\mathrm{H}$, Atamturk $\mathrm{A}$. Therapeutic effects of aquatic exercises on a boy with Duchenne muscular dystrophy. 2018;14(5):877-82.

19. Navarro VT, Granell JC. Design and Validation of a Satisfaction Questionnaire with Sports Programmes in Penitentiaries. Apunt Educ Física y Deport. 2018;(132):94-109.

20. Silvestri M, Crimi E, Oliva S, Senarega D, Tosca MA, Rossi GA, et al. Pulmonary function and airway responsiveness in young competitive swimmers. Pediatr Pulmonol. 2013;48(1):74-80.

21. Lätt $E$, Jürimäe J, Haljaste K, Cicchella A, Purge $P$, Jürimäe T. Physical Development and Swimming Performance During Biological Maturation in Young Female Swimmers. Coll Antropol. 2009;33:117-22.

22. D'Ascenzi F, Pelliccia A, Valentini F, Malandrino A, Natali BM, Barbati R, et al. Training-induced right ventricular remodelling in pre-adolescent endurance athletes: The athlete's heart in children. Int $\mathrm{J}$ Cardiol. 2017;236:270-5.

23. Couto M, Santos P, Silva D, Delgado L, Moreira A. Exhaled breath temperature in elite swimmers: The effects of a training session in adolescents with or without asthma. Pediatr Allergy Immunol. 2015;26:564-70.

24. Weiss MR, McCullagh $P$, Smith AL, Berlant AR. Observational learning and the fearful child: Influence of peer models on swimming skill performance and psychological responses. Res Q Exerc Sport. 1998;69(4):380-94.

25. Mickleborough TD, Stager JM, Chatham K, Lindley MR, lonescu AA Pulmonary adaptations to swim and inspiratory muscle training. Eur J Appl Physiol. 2008;103(6):635-46.

26. Andrade A, Vilarino GT, Sieczkowska SM, Coimbra DR, Steffens R de AK, Vietta GG. Acute effects of physical exercises on the inflammatory markers of patients with fibromyalgia syndrome: A systematic review. J Neuroimmunol. 2018;316(358):40-9.

27. Gonçalves IF, Lavorato VN, Lobato $L$ do VR, Maffia RM, Guedes JM. Nível de aptidão física relacionada à saúde em crianças praticantes de natação. Rev Bras Prescrição e Fisiol do Exerc. 2013;12(2):1052-6.

28. Matias TS, Viana MDS, Kretzer FL, Andrade A. Autodeterminação de adolescentes em diferentes estágios de mudança para o exercício físico. Rev da Educ Física/UEM. 2014;25(2):211-222

29. Voltolini LA, Souza WC, Smolarek C, Lima VA, Costa A, et al. Análise de desempenho do nado crawl por meio da cinemetria. Rev Bras Prescrição e Fisiol do Exerc. 2017;11(69):735-42.

30. Ejnisman B, Andreoli C V, Carrera EF, Abdalla RJ, Cohen M. Lesões músculo-esqueléticas no ombro do atleta: mecanismo de lesão, diagnóstico e retorno à prática esportiva. Rev Bras Ortop. 2001;36(10):389-93. 